\title{
Role of glucose and ketone bodies in the metabolic control of experimental brain cancer
}

\author{
TN Seyfried*,', TM Sanderson', MM El-Abbadi', R McGowan' and P Mukherjee' \\ 'Biology Department, Boston College, Chestnut Hill, MA 02467, USA
}

\begin{abstract}
Brain tumours lack metabolic versatility and are dependent largely on glucose for energy. This contrasts with normal brain tissue that can derive energy from both glucose and ketone bodies. We examined for the first time the potential efficacy of dietary therapies that reduce plasma glucose and elevate ketone bodies in the CT-2A syngeneic malignant mouse astrocytoma. C57BL/6) mice were fed either a standard diet unrestricted (SD-UR), a ketogenic diet unrestricted (KD-UR), the SD restricted to 40\% (SD-R), or the KD restricted to $40 \%$ of the control standard diet (KD-R). Body weights, tumour weights, plasma glucose, $\beta$-hydroxybutyrate $(\beta$-OHB), and insulin-like growth factor I (IGF-I) were measured I 3 days after tumour implantation. CT-2A growth was rapid in both the SDUR and KD-UR groups, but was significantly reduced in both the SD-R and KD-R groups by about $80 \%$. The results indicate that plasma glucose predicts CT-2A growth and that growth is dependent more on the amount than on the origin of dietary calories. Also, restriction of either diet significantly reduced the plasma levels of IGF-I, a biomarker for angiogenesis and tumour progression. Owing to a dependence on plasma glucose, IGF-I was also predictive of CT-2A growth. Ketone bodies are proposed to reduce stromal inflammatory activities, while providing normal brain cells with a nonglycolytic high-energy substrate. Our results in a mouse astrocytoma suggest that malignant brain tumours are potentially manageable with dietary therapies that reduce glucose and elevate ketone bodies.
\end{abstract}

British Journal of Cancer (2003) 89, I375-1382. doi:I0.1038/sj.bjc.660I269 www.bjcancer.com

(c) 2003 Cancer Research UK

Keywords: ketogenic diet; caloric restriction; IGF- I; glioma; angiogenesis; metabolic control

The long-term prognosis remains poor for most patients with malignant brain tumours despite advances in the molecular genetics of cancer and in brain imaging techniques (Shapiro, 1999). Surgical resection followed by radiation is the standard therapy today as it has been for over five decades. Chemotherapy also has had little positive benefit on malignant glioma management and is often associated with adverse effects that diminish quality of life (Shapiro, 1999). Although therapeutic targeting of tumour-associated mutations may be effective in tumour management, most tumour mutations arise as later stage epiphenomena of tissue disorganisation and their involvement with tumour initiation, promotion, or progression has not been conclusively established (Sonnenschein and Soto, 1999, 2000; Seyfried, 2001; Bergstein, 2003). Clearly, alternative therapies are needed that can better manage brain tumours while permitting a decent quality of life.

Metabolic control theory applies principles of bioenergetics for the control or management of complex diseases (Veech et al, 2001; Strohman, 2002; Greene et al, 2003). Since metabolism is a universal process underlying all phenotypes, modification of metabolism can potentially modify phenotype. The theory is based on the idea that compensatory genetic and biochemical pathways regulate the bioenergetic potential of glycolysis, the tricarboxylic acid (TCA) cycle, and the electron transport chain. This produces a flexible and versatile metabolic system that is capable of restoring

\footnotetext{
*Correspondence: Dr TN Seyfried; E-mail: thomas.seyfried@bc.edu
} Received 22 May 2003; revised I 4 July 2003; accepted I 5 July 2003 an orderly adaptive behaviour to widely disordered conditions involving complex gene-environmental interactions (Greenspan, 2001; Strohman, 2002; Greene et al, 2003). As biological chaos underlies the progression of brain tumours (Seyfried, 2001), principles of metabolic control theory may be effective in managing brain cancer.

In 1995, Nebeling et al reported that a ketogenic diet could manage advanced stage malignant astrocytoma in two female paediatric patients (Nebeling et al, 1995). The ketogenic diet (KD) is a high-fat, low-protein, low-carbohydrate diet that has been used for decades to treat patients with refractory epilepsies (Freeman et al, 2000; Todorova et al, 2000; Greene et al, 2003). It was not clear, however, whether $\mathrm{KD}$ controlled paediatric astrocytoma through effects on plasma glucose or ketone bodies since the diet was administered under restricted conditions where blood glucose levels were also reduced (Nebeling et al, 1995). Although the findings with paediatric astrocytoma generated considerable interest in the brain tumour field (Nebeling and Lerner, 1995), no further studies were conducted in humans and no studies have evaluated the efficacy of this dietary therapy in a brain tumour animal model.

While brain cells metabolise glucose for energy under normal physiological conditions, they can metabolise ketone bodies (acetoacetate and $\beta$-hydroxybutyrate) for energy when blood glucose levels decrease as occurs during fasting or caloric restriction (Owen et al, 1967; Clarke and Sokoloff, 1999; Greene et al, 2001, 2003). In contrast to glucose, ketone bodies bypass cytoplasmic glycolysis and directly enter the TCA cycle as acetyl CoA (Sato et al, 1995; Veech et al, 2001). Gliomas and most tumour 
cells, however, lack this metabolic versatility and are largely dependent on glycolytic energy (Fearon et al, 1988; Mies et al, 1990; Oudard et al, 1997; Aronen et al, 2000; Roslin et al, 2003). Defects in ketone body metabolism, the mitochondrial TCA cycle, and electron transport chain systems are thought to underlie the dependence of tumour cells on glycolytic energy (Warberg, 1956; Fredericks and Ramsey, 1978; Tisdale and Brennan, 1983; Lichtor and Dohrmann, 1986; John, 2001). Hence, therapies that exploit the genetic and metabolic weakness of brain tumour cells may be effective in controlling brain cancer.

Dietary caloric restriction (DR) has long been recognised as a natural therapy that improves health, promotes longevity, and significantly reduces the incidence, establishment, and growth of many tumour types (Rous, 1914; Tannenbaum, 1959; Weindruch and Walford, 1988; Birt et al, 1999; Kritchevsky, 1999a; Duan et al, 2003; Koubova and Guarente, 2003). Dietary caloric restriction is produced from a total dietary restriction and differs from acute fasting or starvation in that DR reduces total caloric energy intake without causing anorexia or malnutrition (Tannenbaum, 1959; Mukherjee et al, 1999). We recently showed that a moderate DR of $30-40 \%$ significantly reduced angiogenesis and growth in the CT2A malignant mouse astrocytoma model (Mukherjee et al, 2002). Moreover, DR significantly increased the number of apoptotic cells in the CT-2A tumour. We suggested that DR reduced CT-2A growth through effects on both the tumour cells and on the tumour-associated host cells. Reduced glycolytic energy together with a global downregulation of inflammatory and angiogenic properties of the microenvironment were proposed to underlie the antitumour effects of DR (Mukherjee et al, 1999, 2002).

In this study, we compared the effects of restricted feeding of a standard diet or a KD on the orthotopic growth of the CT-2A brain tumour. While CT-2A growth was rapid with the unrestricted feeding of either diet, a moderate $40 \%$ restriction of either diet significantly reduced growth. Reductions of plasma glucose together with elevations of plasma ketones were associated with the antitumour efficacy of the diets. Also, restriction of either diet significantly reduced the plasma levels of insulin-like growth factor-1 (IGF-1), a biomarker for angiogenesis and tumour progression. Our results in mice suggest that malignant brain tumours may be manageable in part through dietary reduction of glucose and elevation of ketone bodies.

\section{MATERIALS AND METHODS}

\section{Mice}

Mice of the C57BL/6J strain were obtained from Jackson Laboratory (Bar Harbor, ME, USA). The mice were propagated in the animal care facility of Department of Biology of Boston College. Male mice (10-12 weeks of age) were used for the studies and were provided with food under either ad libitum or unrestricted (UR) conditions or under restricted (R) conditions (as below). Water was provided ad libitum to all mice. The animal room was maintained at $22 \pm 1^{\circ} \mathrm{C}$ and cotton nesting pads were provided for additional warmth. All animal experiments were carried out with ethical committee approval in accordance with the National Institutes of Health Guide for the Care and Use of Laboratory Animals and were approved by the Institutional Animal Care Committee. These procedures also met the standards required by the UKCCCR guidelines (Workman et al, 1998).

\section{Brain tumour model}

The syngeneic CT-2A experimental mouse brain tumour used for these studies was the second cerebral tumour (CT-2) generated in our laboratory after implantation of 20-methylcholanthrene into the cerebral cortex of a C57BL/6J mouse according to the procedure of Zimmerman (Zimmerman and Arnold, 1941; Seyfried et al, 1992). Histologically, the CT-2A brain tumour is broadly classified as a poorly differentiated highly malignant anaplastic astrocytoma (Seyfried et al, 1992). The tumour grows orthotopically as a soft, noncohesive, and highly vascularised mass.

\section{Intracerebral tumour implantation}

The CT-2A tumour was implanted into the cerebral cortex of C57BL/6J mice using a trocar as we previously described (Seyfried et al, 1987; Ranes et al, 2001). Briefly, mice were anaesthetised with 2, 2, 2-tribromoethanol intraperitoneally and their heads were shaved and swabbed with $70 \%$ ethyl alcohol under sterile conditions. Small CT-2A tumour pieces from a C57BL/6J donor mouse were matched for size (about $1 \mathrm{~mm}^{3}$ ) based on a grid and were implanted into the right cerebral hemisphere of anaesthetised recipient mice (Ranes et al, 2001). Initiation of tumours from intact tumour pieces is preferable to initiation from cultured cells since the pieces already contain an established microenvironment and vasculature that facilitates rapid tumour establishment and growth (Tannenbaum, 1959). All of the mice recovered completely from the surgical procedure and were returned to their cages when fully active (within $2 \mathrm{~h}$ ). No animals died from either the surgical procedures or from tumour growth. All mice were euthanised prior to tumour morbidity.

\section{Diets}

All mice received PROLAB chow (Agway Inc.,. NY, USA) prior to the experiment. This is the standard diet (SD) and contained a balance of mouse nutritional ingredients. According to the manufacturer's specification, this diet delivers $4.4 \mathrm{kcal} \mathrm{g}^{-1}$ gross energy, where fat, carbohydrate, protein, and fibre comprised 55, 520,225 , and $45 \mathrm{~g} \mathrm{~kg}^{-1}$ of the diet, respectively. The ketogenic diet was obtained from Zeigler Bros., Inc. (Gardners, PA, USA) and also contained a balance of mouse nutritional ingredients. According to the manufacturer's specification, the KD delivers $7.8 \mathrm{kcal} \mathrm{g}^{-1}$ gross energy, where fat, carbohydrate, protein, and fibre comprised 700, 0,128 , and $109 \mathrm{~g} \mathrm{~kg}^{-1}$ of the diet, respectively. The fat in this diet was derived from lard and the diet had a ketogenic ratio (fats : proteins + carbohydrates) of $5.48: 1$.

\section{Dietary restriction}

The mice were group housed prior to the initiation of the experiment and were then housed singly in plastic shoebox cages 1 day before tumour implantation. After tumour implantation, mice were randomly assigned to one of four diet groups that received either: (1) the standard diet fed ad libitum or unrestricted (SDUR), (2) the KD fed ad libitum or unrestricted (KD-UR), (3) the SD restricted to $40 \%$ (SD-R), or (4) the KD restricted to $40 \%$ of the control standard diet (KD-R). Total dietary restriction maintains a constant ratio of nutrients to energy, that is, the average daily food intake $(\mathrm{g})$ for the UR-fed mice was determined every other day and the R-fed mice were given $60 \%$ of the SD-UR group on a daily basis. This ensured that the mice in both $\mathrm{R}$ mouse groups received a similar number of total calories throughout the study. The dietary treatments were initiated $24 \mathrm{~h}$ following tumour implantation and were continued for 13 days. Body weights of all mice were recorded every other day.

\section{Tumour growth}

Intracerebral tumour growth was analysed directly by measuring total tumour dry weight. Tumours were dissected from normal appearing brain tissue, frozen, and then lyophilised to remove water. From our experience, total tumour dry weight is a more accurate measure of tumour growth than total wet weight because 
individual CT-2A tumours can vary in the degree of haemorrhage and oedema.

\section{Measurement of plasma glucose and $\beta$-hydroxybutyrate}

Blood was collected from mice on the last day of the experiment and before tumour resection. The mice were anaesthetised with Isoflurane (Halocarbon), and blood was collected from the heart in heparinised tubes. All mice were fasted for $3 \mathrm{~h}$ before blood collection. The blood was centrifuged at $1600 \mathrm{~g}$ for $10 \mathrm{~min}$, and the plasma was collected and stored at $-80^{\circ} \mathrm{C}$ until assayed. Plasma glucose and $\beta$-hydroxybutyrate $(\beta$-OHB) concentrations were measured spectrophotometrically using the Trinder assay and a UV enzymatic assay, respectively (Sigma, St Louis, MO, USA). We measured only $\beta$-OHB levels because $\beta$-OHB is derived from acetoacetate in the liver and is the major blood ketone body (Krebs et al, 1971; Bhagavan, 2002).

\section{Insulin-like growth factor-1 (IGF-1) analysis}

Plasma IGF-1 concentrations were measured by radioimmunoassay (Nichols Institute Diagnostics, Capistrano, CA, USA) with purified IGF-1 as the standard and controls supplied by kit as we previously described (Mukherjee et al, 1999).

\section{Statistical analysis}

Body weight, food intake, tumour growth and all serum quantitative measurements were analysed by ANOVA followed by Fisher's PLSD to calculate two-sided pairwise comparison among different test groups by use of Statview 5.0. In each figure, $n$ designates the number of individual mice analysed. Error bars in the figures are expressed as $95 \%$ confidence intervals (CIs) $(1.96 \times$ standard error of mean) according to the recommended standards (Streiner, 1996). Linear regression was used to determine the relationships among plasma glucose, $\beta$-OHB, and tumour growth (Lang and Secic, 1997). The regressions are expressed as the slope $\pm 95 \%$ CI.

\section{RESULTS}

No adverse effects were observed in the $40 \%$ R-fed mice that were maintained on either the SD or the KD. Although total body weight was reduced, the R-fed mice were healthy and more physically active than the mice in either UR-fed group. No signs of vitamin or mineral deficiency were observed in the R-fed mice according to standard criteria for mice (Hoag and Dickie, 1968). These findings are consistent with the well-recognised health benefits of mild-tomoderate diet restriction in rodents (Tannenbaum, 1959; Weindruch and Walford, 1988; Keenan et al, 1999). The UR-KD mice also appeared healthy throughout the study, but displayed oily fur and stool discoloration (yellowish). The intestinal tract was also discolored in UR-KD mice upon dissection.

\section{Energy intake}

Energy intake increased significantly about 2 days after intracerebral tumour implantation in both UR-fed groups (Figure 1A). This phenomenon was also observed previously in mice pretreated with DR for 7 days prior to tumour implantation (Mukherjee et al, 2002). This increased energy intake results from hyperphagia (binge eating) that may be associated with cerebral hyperglycolysis following tumour implantation (Gadisseux et al, 1984; Bergsneider et al, 1997; Mukherjee et al, 2002) . In order to maintain a $40 \%$ dietary restriction throughout the study, energy intake was increased in the $\mathrm{R}$-fed mice to compensate for the hyperphagia in the UR-fed mice.
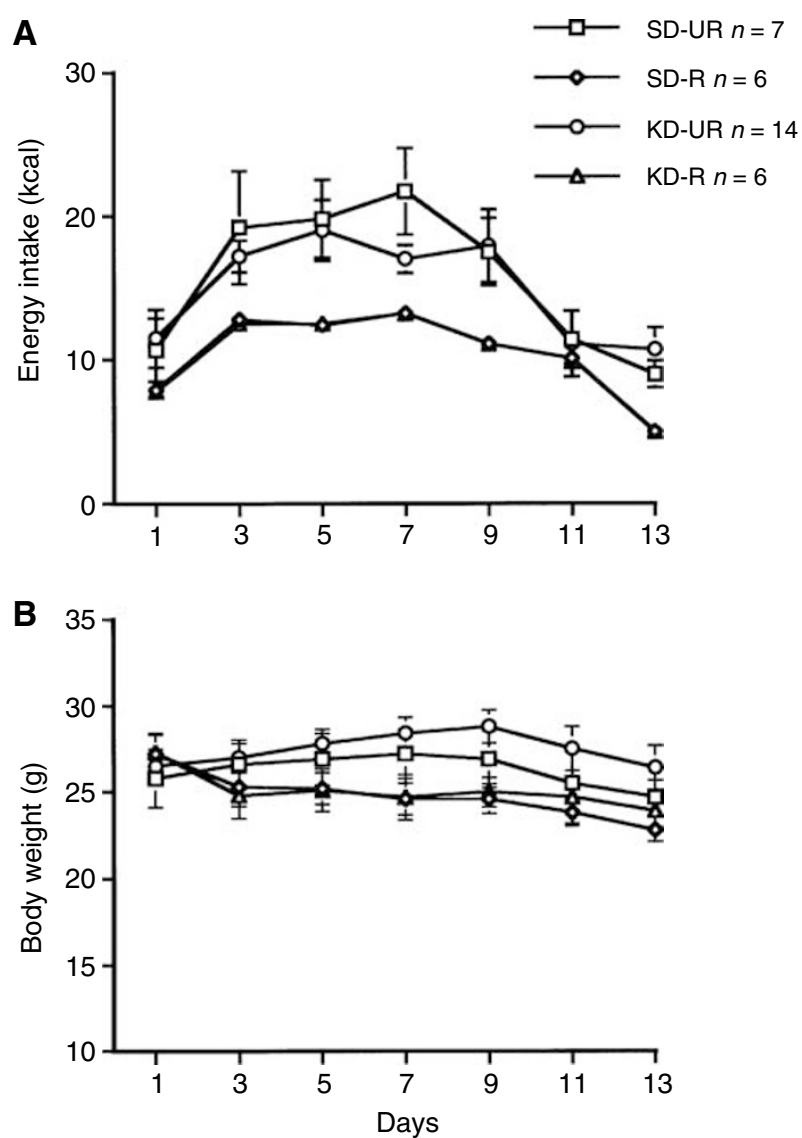

Figure I Energy intake (A) and body weights (B) in male C57BL/6 mice bearing the intracerebral CT-2A brain tumour. Tumours were implanted on day 0 and dietary treatment was started on day I. Values are expressed as means with 95\% confidence intervals, and $n=$ the number of tumour bearing mice examined in each group.

The initial total energy intake of the UR-fed groups was about 11-12 $\mathrm{kcal} \mathrm{day}^{-1}$, but rose to about $20 \mathrm{kcal}_{\text {day }}{ }^{-1}$ during the hyperphagic period (Figure 1A). Energy intake declined in these mice after about day 9 and returned to the initial intake by day 11 . The reduced energy intake beyond day 11 reflected the effects of tumour burden. The total energy intake of the R-fed groups was adjusted to $60 \%$ of that in the UR-SD group throughout the study. Thus, both R-fed groups received similar amounts of total calories despite differences in the caloric origin of the diets.

The hyperphagia in the UR-fed groups was not associated with a significant increase in body weight (Figure 1B). A similar finding was seen in sham-operated mice suggesting that the brain trauma from tumour implantation increased basal metabolic rate. The Rfed mice lost about $10 \%$ of their initial body weight during the first week of treatment and their weights remained significantly lower than those of the UR-fed groups throughout the study $(P<0.001)$. The body weight decline in the R-fed groups after day 9 reflected the body weight decline in the UR-fed groups due to increased tumour burden.

\section{Dietary restrictions reduce intracerebral CT-2A tumour growth}

The intracerebral CT-2A tumour grew rapidly and to a large size in both groups of UR-fed mice (Figure 2). Restricted feeding of either the $\mathrm{SD}$ or the KD significantly reduced tumour growth. The mean tumour dry weight was approximately $86 \%$ less in the SD-R group than in the SD-UR group and was approximately $80 \%$ less in the 


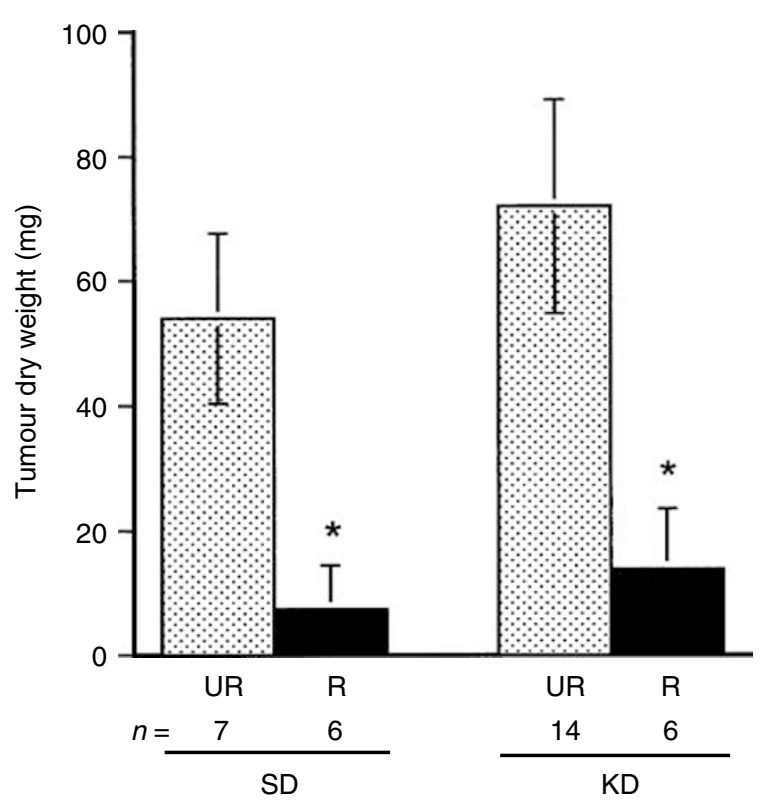

Figure 2 Influence of diet on the intracerebral growth of the CT-2A brain tumour. Dietary treatment was initiated I day after tumour implantation and was continued for 13 days as shown in Figure I. Tumour weights were measured in C57BL/6] mice receiving either the standard diet (SD) or ketogenic diet (KD) under either unrestricted (UR) or restricted (R) feeding. Values are expressed as means with $95 \% \mathrm{Cls}$, and $n=$ the number of mice examined in each group. The dry weights of the tumours in R groups were significantly lower than those in the UR groups at $P<0.01$.

KD-R group than in the KD-UR group (Figure 2). This growth reduction in tumour weight greatly exceeded the reduction in the body weight. It is important to mention that all implanted tumours grew in both the UR- and R-fed groups suggesting that restricted feeding of either the SD or the KD did not prevent tumour 'take' or establishment, but significantly reduced the intracerebral growth of the malignant CT-2A brain tumour.

\section{Influence of diet on plasma glucose and $\beta$-OHB levels}

Plasma glucose levels were significantly lower in the R-fed mouse groups than in the UR-fed groups (Table 1). Plasma glucose levels, however, were similar in both UR-fed mouse groups. These findings are consistent with previous studies in mice that the $\mathrm{KD}$, fed ad libitum, does not significantly alter plasma glucose levels (Todorova et al, 2000). In contrast to glucose levels, $\beta$-OHB levels were two-fold greater in the SD-R mice than in the SD-UR mice (Table 1). Although the ketone levels in the KD-UR and KD-R groups were not significantly different, the ketone levels in both these groups were significantly greater than that in the SD-UR. These findings are consistent with previous studies in mice that $\beta$ $\mathrm{OHB}$ levels are increased under caloric restriction or the KD (Todorova et al, 2000; Greene et al, 2001).

\section{Statistical analysis of plasma glucose levels, $\beta$-OHB levels, and CT-2A tumour growth}

To determine whether blood glucose levels were predictive of blood $\beta$-OHB levels and tumour growth, we analysed the data using simple linear regression. These statistical analyses also included one mouse considered an outlier, that is, a mouse that ate less in the SD-UR group. This mouse also had reduced body weight and plasma glucose. This outlier was considered restricted for caloric intake and similar to the mice in R-fed groups. Although the outlier was excluded from the data presented in Figures 1 and 2, and Table 1, the data from this mouse were included in the
Table I Influence of diet on plasma glucose, $\beta$-OHB, and IGF-I levels in mice bearing the CT-2A intracerebral brain tumour ${ }^{\mathrm{a}}$

\begin{tabular}{|c|c|c|c|c|}
\hline Diet $^{b}$ & Groups $^{c}$ & $\begin{array}{c}\text { Glucose } \\
\left(\mathrm{mmol} \mathrm{I}^{-1}\right)\end{array}$ & $\underset{\left(\mathrm{mmol} \mathbf{l}^{-1}\right)}{\beta \text {-OHB }}$ & $\underset{\left(\mathrm{ng} \mathrm{mI}^{-1}\right)}{\text { IGF-I }}$ \\
\hline \multirow[t]{2}{*}{ SD } & UR & $\begin{array}{c}9.1 \pm 0.9 \\
(7)^{d}\end{array}$ & $\begin{array}{c}0.6 \pm 0.1 \\
(7)\end{array}$ & $208 \pm 25$ \\
\hline & $\mathrm{R}$ & $5.2 \pm 1.1 *$ & $1.4 \pm 0.2 *$ & $117 \pm \frac{36 *}{(6)}$ \\
\hline \multirow[t]{2}{*}{ KD } & UR & $11.4 \pm 1.4$ & $1.0 \pm 0.3$ & $294 \pm \frac{30}{(5)}$ \\
\hline & $\mathrm{R}$ & $5.7 \pm 1.5 *$ & $1.3 \pm 0.6$ & $193 \pm 57 *$ \\
\hline
\end{tabular}

a $V$ alues are expressed as means $\pm 95 \%$ confidence intervals as described in Materials and Methods. ${ }^{b}$ Animals were fed either a standard chow diet (SD) or a ketogenic diet (KD). 'UR (unrestricted feeding) and R (restricted to 60\% of the SD-UR group as described in Materials and Methods). ${ }^{d}$ Numbers in parentheses indicate the number of independent tumour-bearing mice examined in each group. The asterisks indicate that the values of the $R$ groups differed from those of their respective UR groups at $P<0.01$ (analysed by ANOVA, one-way)

regression analyses in order to assess the relationships between glucose, $\beta$-OHB, and tumour growth.

Simple linear regression analysis was used to examine the relationship between plasma glucose levels (the independent or explanatory $X$ variable) and $\beta$-OHB levels (the dependent or response $Y$ variable) for both the $\mathrm{SD}$ and the $\mathrm{KD}$. These variables were identified based on physiological and neurochemical studies showing that plasma glucose levels determine plasma $\beta$-OHB levels during periods of fasting (Clarke and Sokoloff, 1999; Greene et al, 2001). The assumptions of simple linear regression were met according to the established criteria (Lang and Secic, 1997). The slopes of the regression lines were highly significant $(P<0.01)$ and showed that plasma $\beta$-OHB levels increased as glucose levels decreased for the SD $(-0.143 \pm 0.074 ; t=-4.22 ; Y=2.03-0.143 X$, $n=14)$ and for the $\mathrm{KD}(-0.099 \pm 0.063 ; t=-3.26 ; Y=2.028-$ $0.099 X, n=20)$. The coefficient of determination $\left(r^{2}\right)$ was greater in the SD group (0.598) than in the KD group (0.384). This is expected since ketone levels are higher in the KD-UR mice than in the SD-UR mice and this would reduce the association. Viewed together, these findings indicate that plasma $\beta$-OHB levels are dependent on plasma glucose levels and that glucose levels predict $\beta$-OHB levels in both dietary groups.

We next used linear regression to analyse the association between plasma glucose (the $X$ variable) and CT-2A growth (the $Y$ variable) for both dietary groups combined (Figure 3 ). The slope of the regression line was highly significant $(7.96+2.75 ; t=5.90$; $Y=-23.84 \pm 7.96 \mathrm{X}, n=34$ ) (Figure 3). Thus, plasma glucose levels were highly significant in predicting CT-2A growth.

Since the above linear regression indicated that $\beta$-OHB levels were dependent on glucose levels, the slope was significant for the association between CT-2A growth and $\beta$-OHB levels in the SD group $\left(r^{2}=0.637\right)$, but not for the KD group $\left(r^{2}=0.085\right)$. Hence, no association was seen between CT-2A growth and blood ketone levels (Figure 2 and Table 1 ) indicating that ketone elevation alone is unable to reduce growth.

\section{Plasma IGF-1}

Circulating IGF-1 levels were significantly lower in each R-fed mouse group than in the respective UR-fed group (Table 1). Changes in plasma IGF-1 levels were also associated with changes in plasma glucose levels (Table 1). Indeed, linear regression analysis indicated that plasma glucose is predictive of plasma IGF1 levels (Figure 4A). The slope of the regression line was significant and showed that IGF-1 decreased as glucose levels decreased $(15.42 \pm 11.68 ; t=2.74 ; Y=92.20+15.42 X)$. Since plasma glucose 


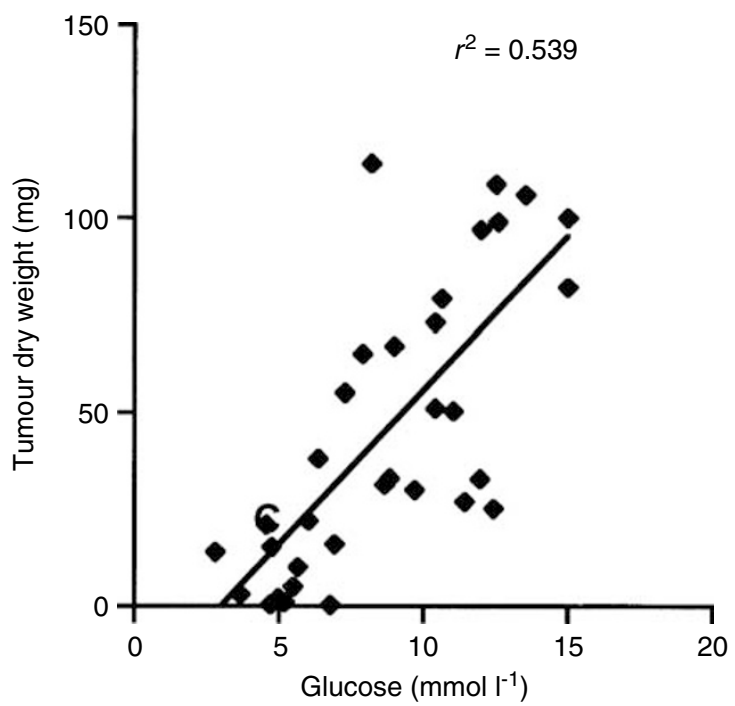

Figure 3 Linear regression analysis of plasma glucose and CT-2Atumour growth in mice from both the SD and KD dietary groups combined $(n=34)$. These analyses included the values for plasma glucose and tumour growth of individual mice from both the UR- and R-fed groups. The linear regression was highly significant at $* P<0.001$.

levels are predictive of both tumour growth and IGF-1 levels, we next analysed the relationship between plasma IGF-1 levels and CT-2A tumour growth (Figure 4B). The slope of this regression line was also significant and showed that CT-2A tumour growth decreased as IGF-1 levels decreased $(0.20 \pm 0.02 ; t=2.43$; $Y=-6.84+0.20 X)$. These findings indicate that plasma IGF-1, like plasma glucose, is predictive of CT-2A growth.

\section{DISCUSSION}

Normal mammalian brain cells are metabolically versatile and capable of deriving energy from glucose and ketone bodies (Greene et al, 2003). Although the levels of glucose and ketones in brain are proportional to their levels in blood, the adult brain does not usually metabolise ketones for energy under normal physiological conditions (Clarke and Sokoloff, 1999; Laterra et al, 1999; Guzman and Blazquez, 2001; Bhagavan, 2002). The brain will, however, actively metabolise ketone bodies for energy if blood glucose levels are reduced. We recently showed that circulating ketone levels are inversely related to circulating glucose levels in calorie restricted epileptic mice (Greene et al, 2001). The results of the present study support these previous findings. Ketone bodies bypass cytoplasmic glycolysis and directly enter the TCA cycle as acetyl CoA (Nehlig and Pereira de Vasconcelos, 1993; Sato et al, 1995; Kashiwaya et al, 2000; Veech et al, 2001). This causes significant increases in the TCA cycle metabolites and improves physiological performance through an increase in the energy of ATP hydrolysis (Sato et al, 1995; Veech et al, 2001).

We suggest that the metabolic mechanism by which moderate DR inhibits CT-2A brain tumour growth involves a failure to utilise alternative energy substrates. Most tumours including primary brain tumours actively consume glucose and are largely dependent on glycolysis for energy (Warberg, 1956; Galarraga et al, 1986; Mies et al, 1990; Oudard et al, 1997; Roslin et al, 2003). Mitochondrial defects and an inability to metabolise ketone bodies are thought to be responsible for the dependence of tumour cells on glycolytic energy (Warberg, 1956; Fredericks and Ramsey, 1978; Pedersen, 1978; Tisdale and Brennan, 1983; Lichtor and Dohrmann, 1986; Oudard et al, 1997; John, 2001). The flexibility and versatility of gene-linked metabolic network interactions in
A

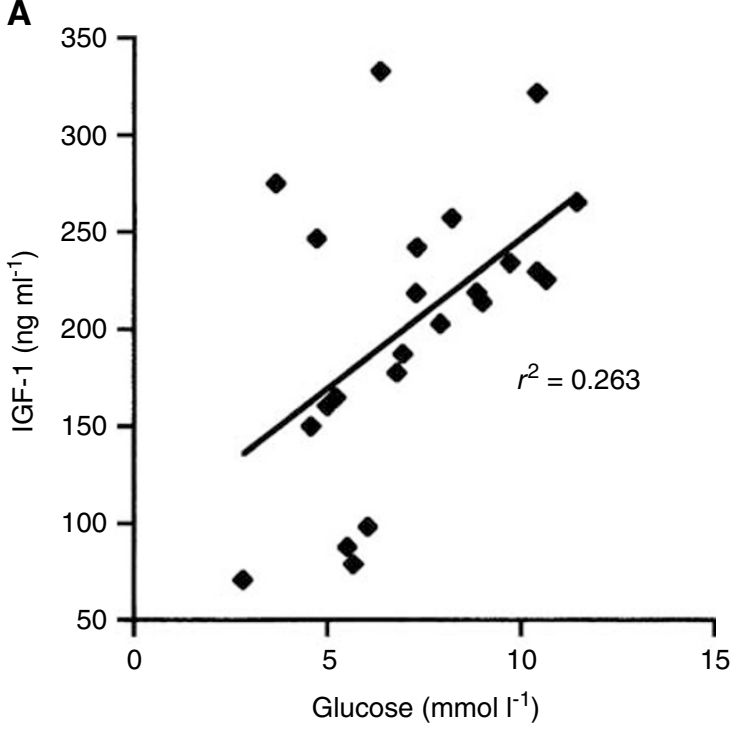

B

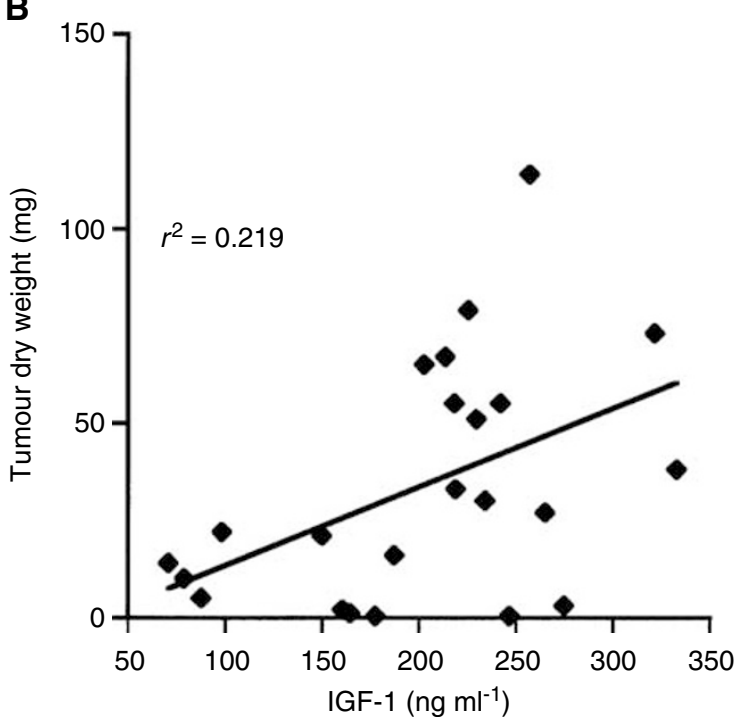

Figure 4 Linear regression analysis of plasma glucose and IGF-I levels (A), and plasma IGF-I levels and CT-2A-tumour growth (B) in mice fed with both the SD and $\mathrm{KD}(n=23)$. These analyses included the values for plasma glucose and $\beta-\mathrm{OHB}$ levels of individual mice from both the UR- and $\mathrm{R}$-fed groups. The linear regressions were highly significant at $P<0.0 \mathrm{I}$.

response to alterations in nutritional environment underlies metabolic control theory (Greenspan, 2001; Veech et al, 2001; Strohman, 2002; Greene et al, 2003). As DR inhibits glycolysis, DR would force cells to switch from glucose to alternative noncarbohydrate energy metabolites, for example, ketones (Greene et al, 2003; Hagopian et al, 2003). While this switch occurs readily in normal cells, the switch is more difficult for tumour cells due to their accumulated genetic defects. Our results in the CT-2A astrocytoma support the feasibility of metabolic control for the management of brain cancer.

CT-2A growth reduction was associated with reduced blood glucose levels. Indeed, linear regression analysis showed that blood glucose levels were predictive of CT-2A growth. Although blood ketone levels were elevated under restriction of either diet, elevated ketone levels alone could not account for reduced tumour growth because tumour growth was rapid in the UR-KD group despite the presence of high ketone levels. These findings support the previous observations of Fearon et al (1985), who showed that the failure of a KD to restrict growth of the Walker 256 rat tumour resulted from 
the failure of ketosis to reduce glucose availability. Moreover, reduced blood glucose was associated with the management of advanced stage malignant astrocytoma in two female paediatric patients with the KD (Nebeling and Lerner, 1995; Nebeling et al, 1995). Hence, reduced glucose is a key factor in the metabolic control of the mouse CT-2A tumour and possibly human paediatric astrocytoma.

If the antitumour effects of restricted caloric intake are associated with reduced glucose levels and glycolytic energy, a question arises as to what role elevated ketone levels might have in CT-2A management. We suggest that ketone body metabolism, while providing normal brain cells with an alternative high-energy substrate, also reduces the inflammatory activities of tumourassociated host cells (stromal cells). Ketone body metabolism reduces oxygen free radicals, enhances tolerance to hypoxia, and may prevent organ dysfunction from inflammatory processes (Kirsch and D'Alecy, 1984; Yu et al, 1990; Marcondes et al, 2001; Veech et al, 2001; Sharman et al, 2002). Indeed, Dong et al (1998) reported that moderate caloric restriction could reduce the proinflammatory properties of macrophages while enhancing their phagocytic function. Activated macrophages also contribute to tumour angiogenesis that is reduced under DR (Mukherjee et al, 1999, 2002). Uncoupling the detrimental inflammatory activities of macrophages from their potentially beneficial phagocytic activities is considered important for the eventual management of brain cancer (Seyfried, 2001). Hence, a shift in energy metabolism from glucose to ketones will enhance the bioenergetic potential of normal brain cells on the one hand while reducing tumour cell growth and tumour inflammatory properties on the other hand.

Insulin-like growth facor-1 is considered a biomarker for angiogenesis and tumour progression (Kari et al, 1999; Mukherjee et al, 1999; Yu and Rohan, 2000). Besides predicting CT-2A growth, we found that plasma glucose also predicts plasma IGF-1 levels. These observations agree with previous studies that glucose regulates IGF-1 expression (Straus and Burke, 1995; Wang et al, 2000). We recently presented histological evidence that moderate dietary restriction significantly reduces vascularity (angiogenesis) in the CT-2A brain tumour (Mukherjee et al, 2002). Our findings that moderate restriction of either the $\mathrm{SD}$ or the $\mathrm{KD}$ reduced circulating IGF-1 levels support further the antiangiogenic effects of DR. Since plasma IGF-1 and glucose are both predictive of CT2A tumour growth, we suggest that either glucose or IGF-1 may be useful biomarkers for predicting the effects of DR on brain tumour growth and angiogenesis.

In addition to reducing angiogenesis, moderate $\mathrm{DR}$ also enhances apoptosis in the CT-2A tumour and in prostate cancer (Mukherjee et al, 1999, 2002). This is the interesting in light of recent findings that the glycolysis inhibitor 2-deoxy-D-glucose or glucose deprivation enhances apoptosis in human breast and lung cancer cells (Lee et al, 1997; Shim et al, 1998; Aft et al, 2002). Since $\mathrm{DR}$ also reduces blood glucose levels, it is possible that DR reduces CT-2A tumour growth through glucose-dependent apoptotic pathways similar to those seen in other tumours (Shim et al,

\section{REFERENCES}

Aft RL, Zhang FW, Gius D (2002) Evaluation of 2-deoxy-D-glucose as a chemotherapeutic agent: mechanism of cell death. Br J Cancer 87: 805 812

Aronen HJ, Pardo FS, Kennedy DN, Belliveau JW, Packard SD, Hsu DW, Hochberg FH, Fischman AJ, Rosen BR (2000) High microvascular blood volume is associated with high glucose uptake and tumor angiogenesis in human gliomas. Clin Cancer Res 6: 2189-2200

Bergsneider M, Hovda DA, Shalmon E, Kelly DF, Vespa PM, Martin NA, Phelps ME, McArthur DL, Caron MJ, Kraus JF, Becker DP (1997) Cerebral hyperglycolysis following severe traumatic brain injury in
1998; Aft et al, 2002). Further studies will be needed to test this hypothesis.

In contrast to the situation with prostate cancer and other nonneural cancers (Tannenbaum, 1959; Bosland et al, 1999; Kritchevsky, 1999b; Mukherjee et al, 1999), little is known about the influence of diet on the progression of brain cancer. We found that orthotopic growth of the CT-2A brain tumour was similarly rapid during the unrestricted feeding of either a standard laboratory diet or a high-fat KD suggesting that a high-fat diet does not significantly enhance growth over that of a standard diet fed ad libitum. On the other hand, CT-2A growth was significantly reduced when either diet was restricted to $60 \%$ of the control diet. These findings indicate that orthotopic CT-2A brain tumour growth, like prostate tumour growth, is influenced more by the amount of dietary calories than by the origin or source of the calories (Tannenbaum, 1959; Mukherjee et al, 1999). Hence, diet and lifestyle may influence the progression of brain cancer.

An issue not addressed in this study is whether restricted feeding of either the SD or the KD would extend the survival of the CT-2A tumour-bearing mice. Survival studies are difficult with this rapidly growing brain tumour model because intracranial pressure, which often causes morbidity in brain tumour patients, is relieved due to skull expansion or tumour growth through the implantation burr hole (Mukherjee et al, 2002). This makes estimates of longevity capricious. Although the overall health was better in the R-fed mice than in the UR-fed mice, further studies will be necessary to determine if DR would extend survival.

In summary, our results suggest that experimental brain cancer is manageable through principles of metabolic control where plasma glucose levels are reduced and ketone body levels are elevated. Dietary energy restriction reduces tumour growth through effects on angiogenesis, apoptosis, and inflammation. Moreover, this dietary therapy may be effective for brain cancer management in humans and can be designed according to established standards (Nebeling and Lerner, 1995; Freeman et al, 2000). Body weight reductions of $10-12 \%$ should not adversely affect development or other functions in children as long as rate of weight gain is maintained (Nebeling and Lerner, 1995; Freeman et al, 2000; Greene et al, 2001). We also previously suggested that DR could reduce tumour-derived levels of procachexic factors by reducing tumour size (Mukherjee et al, 2002). Dietary caloric restriction may also be effective against recurrent human gliomas or could be used as an adjuvant with radiation or chemotherapy. We contend that dietary therapy may improve the clinical outcome of brain tumour patients and enhance their quality of life.

\section{ACKNOWLEDGEMENTS}

This work was supported in part by NIH Grant (HD39722), the Boston College Research Expense Fund, and a grant from the American Institute of Cancer Research (AICR). humans: a positron emission tomography study. J Neurosurg 86: $241-251$

Bergstein I (2003) A non-Darwinian role for mutagenesis in stem cellderived cancers. Mol Carcinogen 36: 1-5

Bhagavan NV (2002) Medical Biochemistry. Harcourt: New York

Birt DF, Yaktine A, Duysen E (1999) Glucocorticoid mediation of dietary energy restriction inhibition of mouse skin carcinogenesis. J Nutr 129: $571 \mathrm{~S}-574 \mathrm{~S}$

Bosland MC, Oakley-Girvan I, Whittemore AS (1999) Dietary fat, calories, and prostate cancer risk. J Natl Cancer Inst 91: 489-491 
Clarke DD, Sokoloff L (1999) Circulation and energy metabolism in the brain. In Basic Neurochemistry, Siegel GJ, Agranoff BW, Albers RW, Fisher SK, Uhler MD (eds) pp 637-669. New York: Lippincott-Raven

Dong W, Selgrade MK, Gilmour IM, Lange RW, Park P, Luster MI, Kari FW (1998) Altered alveolar macrophage function in calorie-restricted rats. Am J Respir Cell Mol Biol 19: 462-469

Duan W, Guo Z, Jiang H, Ware M, Li XJ, Mattson MP (2003) Dietary restriction normalizes glucose metabolism and BDNF levels, slows disease progression, and increases survival in huntingtin mutant mice. Proc Natl Acad Sci USA 100: 2911-2916

Fearon KC, Borland W, Preston T, Tisdale MJ, Shenkin A, Calman KC (1988) Cancer cachexia: influence of systemic ketosis on substrate levels and nitrogen metabolism. Am J Clin Nutr 47: 42-48

Fearon KC, Tisdale MJ, Preston T, Plumb JA, Calman KC (1985) Failure of systemic ketosis to control cachexia and the growth rate of the Walker 256 carcinosarcoma in rats. Br J Cancer 52: 87-92

Fredericks M, Ramsey RB (1978) 3-Oxo acid coenzyme A transferase activity in brain and tumors of the nervous system. J Neurochem 31: $1529-1531$

Freeman JM, Freeman JB, Kelly MT (2000) The Ketogenic Diet: A Treatment for Epilepsy. New York: Demos

Gadisseux P, Ward JD, Young HF, Becker DP (1984) Nutrition and the neurosurgical patient. J Neurosurg 60: 219-232

Galarraga J, Loreck DJ, Graham JF, DeLaPaz RL, Smith BH, Hallgren D, Cummins CJ (1986) Glucose metabolism in human gliomas: correspondence of in situ and in vitro metabolic rates and altered energy metabolism. Metab Brain Dis 1: 279-291

Greene AE, Todorova MT, McGowan R, Seyfried TN (2001) Caloric restriction inhibits seizure susceptibility in epileptic EL mice by reducing blood glucose. Epilepsia 42: 1371-1378

Greene AE, Todorova MM, Seyfried TN (2003) Perspectives on the metabolic management of epilepsy through dietary reduction of glucose and elevation of ketone bodies. J Neurochem, 86: 529-537

Greenspan RJ (2001) The flexible genome. Nat Rev Genet 2: 383-387

Guzman M, Blazquez C (2001) Is there an astrocyte-neuron ketone body shuttle? Trends Endocrinol Metab 12: 169-173

Hagopian K, Ramsey JJ, Weindruch R (2003) Influence of age and caloric restriction on liver glycolytic enzyme activities and metabolite concentrations in mice. Exp Gerontol 38: 253-266

Hoag WG, Dickie MM (1968) Nutrition. In Biology of the Laboratory Mouse, Green EL (ed). New York: Dover

John AP (2001) Dysfunctional mitochondria, not oxygen insufficiency, cause cancer cells to produce inordinate amounts of lactic acid: the impact of this on the treatment of cancer. Med Hypotheses 57: $429-431$

Kari FW, Dunn SE, French JE, Barrett JC (1999) Roles for insulin-like growth factor-1 in mediating the anti-carcinogenic effects of caloric restriction. J Nutr Health Aging 3: $92-101$

Kashiwaya Y, Takeshima T, Mori N, Nakashima K, Clarke K, Veech RL (2000) D-beta-hydroxybutyrate protects neurons in models of Alzheimer's and Parkinson's disease. Proc Natl Acad Sci USA 97: $5440-5444$

Keenan KP, Ballam GC, Soper KA, Laroque P, Coleman JB, Dixit R (1999) Diet, caloric restriction, and the rodent bioassay. Toxicol Sci 52: 24-34

Kirsch JR, D'Alecy LG (1984) Hypoxia induced preferential ketone utilization by rat brain slices. Stroke 15: $319-323$

Koubova J, Guarente L (2003) How does calorie restriction work? Genes Dev 17: $313-321$

Krebs HA, Williamson DH, Bates MW, Page MA, Hawkins RA (1971) The role of ketone bodies in caloric homeostasis. Adv Enzyme Reg 9: 387 - 409

Kritchevsky D (1999a) Caloric restriction and experimental carcinogenesis. Toxicol Sci 52: $13-16$

Kritchevsky D (1999b) Fundamentals of nutrition: applications to cancer research. In Nutritional Oncology, Heber D, Blackburn GL, Go VLW (eds) pp 5-10. Boston: Academic Press

Lang TA, Secic M (1997) How to Report Statistics in Medicine. Philadelphia: American College of Physicians

Laterra J, Keep R, Betz AL, Goldstein GW (1999) Blood-braincerebrospinal fluid barriers. In Basic Neurochemistry: Molecular, Cellular and Medical Aspects, Siegel GJ, Agranoff BW, Albers RW, Fisher SK, Uhler MD (eds) pp 671-689. New York: Lippincott-Raven

Lee YJ, Galoforo SS, Berns CM, Tong WP, Kim HR, Corry PM (1997) Glucose deprivation-induced cytotoxicity in drug resistant human breast carcinoma MCF-7/ADR cells: role of c-myc and bcl-2 in apoptotic cell death. J Cell Sci 110(Part 5): 681-686
Lichtor T, Dohrmann GJ (1986) Respiratory patterns in human brain tumors. Neurosurgery 19: $896-899$

Marcondes S, Turko IV, Murad F (2001) Nitration of succinyl-CoA:3oxoacid CoA-transferase in rats after endotoxin administration. Proc Natl Acad Sci USA 98: 7146-7151

Mies G, Paschen W, Ebhardt G, Hossmann KA (1990) Relationship between of blood flow, glucose metabolism, protein synthesis, glucose and ATP content in experimentally induced glioma (RG1 2.2) of rat brain. J Neurooncol 9: 17-28

Mukherjee P, El-Abbadi MM, Kasperzyk JL, Ranes MK, Seyfried TN (2002) Dietary restriction reduces angiogenesis and growth in an orthotopic mouse brain tumour model. Br J Cancer 86: 1615-1621

Mukherjee P, Sotnikov AV, Mangian HJ, Zhou JR, Visek WJ, Clinton SK (1999) Energy intake and prostate tumor growth, angiogenesis, and vascular endothelial growth factor expression. J Natl Cancer Inst 91: $512-523$

Nebeling LC, Lerner E (1995) Implementing a ketogenic diet based on medium-chain triglyceride oil in pediatric patients with cancer. $\mathrm{J} \mathrm{Am}$ Diet Assoc 95: 693-697

Nebeling LC, Miraldi F, Shurin SB, Lerner E (1995) Effects of a ketogenic diet on tumor metabolism and nutritional status in pediatric oncology patients: two case reports. J Am Coll Nutr 14: 202-208

Nehlig A, Pereira de Vasconcelos A (1993) Glucose and ketone body utilization by the brain of neonatal rats. Prog Neurobiol 40: $163-221$

Oudard S, Boitier E, Miccoli L, Rousset S, Dutrillaux B, Poupon MF (1997) Gliomas are driven by glycolysis: putative roles of hexokinase, oxidative phosphorylation and mitochondrial ultrastructure. Anticancer Res 17: $1903-1911$

Owen OE, Morgan AP, Kemp HG, Sullivan JM, Herrera MG, Cahill Jr GF (1967) Brain metabolism during fasting. J Clin Invest 46: 1589-1595

Pedersen PL (1978) Tumor mitochondria and the bioenergetics of cancer cells. Prog Exp Tumor Res 22: 190-274

Ranes MK, El-Abbadi M, Manfredi MG, Mukherjee P, Platt FM, Seyfried TN (2001) $N$-Butyldeoxynojirimycin reduces growth and ganglioside content of experimental mouse brain tumours. $\mathrm{Br} J$ Cancer 84: $1107-1114$

Roslin M, Henriksson R, Bergstrom P, Ungerstedt U, Bergenheim AT (2003) Baseline levels of glucose metabolites, glutamate and glycerol in malignant glioma assessed by stereotactic microdialysis. J Neuro-oncol 61: $151-160$

Rous P (1914) The influence of diet on transplanted and spontaneous mouse tumors. J Exp Med 20: 433-451

Sato K, Kashiwaya Y, Keon CA, Tsuchiya N, King MT, Radda GK, Chance B, Clarke K, Veech RL (1995) Insulin, ketone bodies, and mitochondrial energy transduction. FASEB J 9: 651-658

Seyfried TN (2001) Perspectives on brain tumor formation involving macrophages, glia, and neural stem cells. Perspect Biol Med 44: $263-282$

Seyfried TN, El-Abbadi M, Roy ML (1992) Ganglioside distribution in murine neural tumors. Mol Chem Neuropathol 17: 147-167

Seyfried TN, Yu RK, Saito M, Albert M (1987) Ganglioside composition of an experimental mouse brain tumor. Cancer Res 47: 3538-3542

Shapiro WR (1999) Current therapy for brain tumors: back to the future. Arch Neurol 56: 429-432

Sharman MJ, Kraemer WJ, Love DM, Avery NG, Gomez AL, Scheett TP, Volek JS (2002) A ketogenic diet favorably affects serum biomarkers for cardiovascular disease in normal-weight men. J Nutr 132: 1879-1885

Shim H, Chun YS, Lewis BC, Dang CV (1998) A unique glucose-dependent apoptotic pathway induced by c-Myc. Proc Natl Acad Sci USA 95: $1511-1516$

Sonnenschein C, Soto AM (1999) The Society of Cells: Cancer and the Control of Cell Proliferation. New York: Springer-Verlag

Sonnenschein C, Soto AM (2000) Somatic mutation theory of carcinogenesis: why it should be dropped and replaced. Mol Carcinogen 29: $205-211$

Straus DS, Burke EJ (1995) Glucose stimulates IGF-I gene expression in C6 glioma cells. Endocrinology 136: $365-368$

Streiner DL (1996) Maintaining standards: Differences between the standard deviation and standard error, and when to use each. Can J Psychiatry 41: 498-502

Strohman R (2002) Maneuvering in the complex path from genotype to phenotype. Science 296: $701-703$

Tannenbaum A (1959) Nutrition and cancer. In Physiopathology of Cancer, Homburger F (ed) pp 517-562. New York: Paul B Hober 
Tisdale MJ, Brennan RA (1983) Loss of acetoacetate coenzyme A transferase activity in tumours of peripheral tissues. $\mathrm{Br} J$ Cancer 47: $293-297$

Todorova MT, Tandon P, Madore RA, Stafstrom CE, Seyfried TN (2000) The ketogenic diet inhibits epileptogenesis in EL mice: a genetic model for idiopathic epilepsy. Epilepsia 41: 933-940

Veech RL, Chance B, Kashiwaya Y, Lardy HA, Cahill Jr GF (2001) Ketone bodies, potential therapeutic uses. IUBMB Life 51: $241-247$

Wang L, Yang H, Adamo ML (2000) Glucose starvation reduces IGF-I mRNA in tumor cells: evidence for an effect on mRNA stability. Biochem Biophys Res Commun 269: $336-346$

Warberg O (1956) On the origin of cancer cells. Science 123: $309-$ 314

Weindruch R, Walford RL (1988) The Retardation of Aging and Disease by Dietary Restriction. Springfield, IL: Thomas

Workman P, Twentyman P, Balkwill F, Balmain A, Chaplin D, Double J, Embleton J, Newell D, Raymond R, Stables J, Stephens T, Wallace J (1998) United Kingdom Co-ordinating Committee on Cancer Research (UKCCCR) Guidelines for the Welfare of Animals in Experimental Neoplasia (Second Edition). Br J Cancer 77: 1-10

Yu BP, Lee DW, Marler CG, Choi JH (1990) Mechanism of food restriction: protection of cellular homeostasis. Proc Soc Exp Biol Med 193: 13 - 15

$\mathrm{Yu}$ H, Rohan T (2000) Role of the insulin-like growth factor family in cancer development and progression. J Natl Cancer Inst 92: 1472-1489

Zimmerman HM, Arnold H (1941) Experimental brain tumors: I. tumors produced with methylcholanthrene. Cancer Res 1: 919-938 